\title{
Diffusion and Random Chain Scission
}

\author{
H. H. G. JeLLINEK* \\ Institute of Space and Aeronautical Science, \\ The University of Tokyo, Komaba, Meguro-ku, Tokyo, Japan.
}

(Received June 30, 1972)

\begin{abstract}
Chain scission has been discussed as a process involving medium cages and diffusion of polymer fragments out of such cages. Two types of processes can be distinguished: completion of potential chain scission by segmental diffusion of chain ends and translational diffusion, respectively. The first will be operative for chain scission in solution with flexible polymer chains or for bulk scission. The second can be significant for stiff chains in solution. In both cases chain scission rate constants should be proportional to the reciprocal local medium viscosity. Segmental diffusion should show a dependence on reciprocal chain length for relatively short chains whereas translational diffusion should always be a strong function of reciprocal chain length. Data found in the literature concerning chain scission has been discussed in the light of such cage processes.
\end{abstract}

KEY WORDS Chain Scission / Degradation / Diffusion /

Scission of main chain links can be considered to proceed via medium cages. As long as chain fragments or chain ends are in such cages, the process can still be reversed during the time which is needed for the species to diffuse out of cages. Only if such diffusion has taken place, scission is completed.

Different modes of chain scission can be envisaged according to the chemical nature of the polymer molecules involved in such processes. Some will be ruptured forming polymer radicals while others will be severed while disproportionation takes place practically simultaneously with scission. In the latter instance, conditions can be imagined where the chain break is irreversible even before chains have diffused out of the cage. Here, cage formation does not play any role. However, in most cases whether scission takes place forming radicals or chain fragments by disproportionation, reversal of the process is still possible as long as the relevant species are located in a medium cage or sphere of action of a definite radius whose magnitude depends on the polymer system and on reaction conditions. Chain scission can be considered as the reversal of chain termination in polymer-

* Permanent address: Department of Chemistry, Clarkson College of Technology, Potsdam, New York 13676, U.S.A. ization reactions.

Chain scission may take place either in solution or in bulk. In the latter case, morphology, i.e., percentage of crystallinity of the polymer will play a role. Chain scission in solution can occur either in the presence of solvents which can terminate polymer radicals by a pseudo-first-order reaction, or which can initiate chain scission by transfer reactions. The latter can also occur with bulk polymers; transfer in the latter instance would proceed via cages.

In addition to the above reactions, depropagation may take place from newly formed chain ends producing small molecules such as monomer. Such reactions are characterized by a number-average kinetic chain or zip length. If termination proceeds by mutual combination only, then the number-average polymer chain length_— starting with a homodispersed sample - is practically unchanged; however, the sample will not remain monodisperse. In case a depropagation reaction is also operative, the rate of monomer formation will be governed by the reversible chain scission reaction which proceeds via cages and the number-average chain length will show a slow decrease with time.

Thus there are a multitude of possible reactions and it is not intended to discuss here all 
the various combinations. Only some of the possibilities will be treated in a simple way as far as diffusion processes are concerned. However, such treatment will point out the main characteristics of such reactions. A more detailed and refined treatment has to await more experimental data in this field.

There are two possibilities by which chain scission can be completed, i.e., by translational diffusion of polymer fragments or by segmental diffusion of activated or radical polymer chain ends out of cages. Essentially these are reversals of mutual termination in polymerization reactions. It should be noted that in the case of the termination step in polymerization, these two diffusion processes are analogous to two reactions in series (segmental diffusion can only become effective after the radicals have approached sufficiently near by translational diffusion), whereas for the reverse process, i.e., scission, these processes correspond to parallel reactions (either translational or segmental diffusion separates the radical chain ends, whichever is the faster). In the first case, the slowest reaction dominates the whole process, while in the case of chain scission, the faster reaction makes the major contribution to the overall process.

Chain scission in dilute solution will be discussed first. A completely flexible chain in a solvent will practically be in random coil conformation. If a chain break occurs in a sufficiently long chain molecule, then this coiled up molecule can still be considered to move as one unit by translational diffusion due to intramolecular entanglements for an appreciable period of time.

If this would be the only possible diffusion process, chain scission would practically always be reversed; however, the new chain ends have a definite segment mobility and have the possibility to diffuse sufficiently quickly out of a segmental cage or sphere of action before recombination takes place (see Figure 1). This mobility can be treated in an approximate way as Brownian movement. However, such movement is not completely free but the rotation of segment is hindered and needs a definite energy of activation depending on the chemical nature

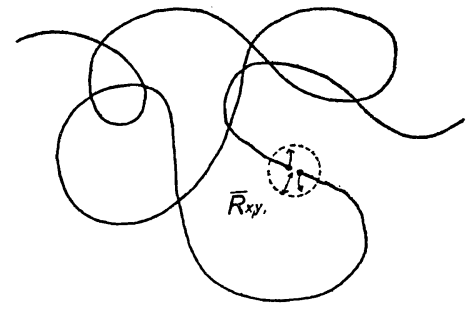

Figure 1. Chain scission of a sufficiently long flexible chain molecule in solution $\left(\bar{R}_{x y} \sim\right.$ average segment cage radius).

of the polymer chains.

This hindrance to free rotation can be taken care of by multiplying segmental diffusion by a factor $\beta<1$ or $\beta=\mathrm{e}^{-B^{\prime}}=k^{\prime} \mathrm{e}^{-B}$.

If one of the chain end segments in the cage of radius $R_{x, y}$ is considered to remain stationary while the other chain end segment carries out unhindered Brownian movement in three dimensions, then it suffers a mean displacement $\bar{R}_{x, y}$ relative to the stationary chain end during time $t$. Hence,

$$
\bar{R}_{x, y}^{2}=6 D_{x, y} t
$$

Here $D_{x, y}=D_{x}+D_{y}$ is the diffusion coefficient of chain end $x$ (or $y$ ) relative to $y$ (or $x$ ); $D_{x}$ and $D_{y}$ are the diffusion coefficients for the respective chain ends (for chain scission, the fragments of each scission can be considered to be, on the average, of equal length). As a large number of radical chain ends are involved in the whole reaction system, the parameters in eq 1 can be considered as statistical averages.

The time $t$ can be considered as the average life time of a newly formed chain end in a cage. This time also represents the half life of a first order reaction. Hence,

$$
k_{s}=\frac{\ln 2}{t}
$$

or

$$
k_{s}=\frac{6 D_{x, y} \ln 2}{\bar{R}_{x, y}^{2}}=\frac{4.14 D_{x, y}}{\bar{R}_{x, y}^{2}}
$$

$k_{s}$ is the rate constant for chain scission. However, as pointed out above, eq 1 has to be multiplied by $k^{\prime} \mathrm{e}^{-B}$ because of hindered rotation of segments. $B$ can be considered to contain 


\section{Diffusion and Random Chain Scission}

the activation energy for rotation, i.e., $B=E / R T$. Thus,

$$
k_{s}^{\prime}=\frac{4.14 D_{x, y} k^{\prime} \mathrm{e}^{-B}}{\bar{R}_{x, y}^{2}}
$$

$B$ can be calculated in principle on the basis of statistical considerations of segment mobility. However, this will not be attempted here; $D_{x, y}$, if based on the Stokes-Einstein relation, is inversely proportional to the local medium viscosity. $\bar{R}_{x, y}$ will be practically independent of polymer chain length as long as this length is large.

An intermediate case will arise if the chain happens not to be long enough. In this instance the contributions from translational and segmental diffusion, respectively, may be of similar magnitude and chain scission may become dependent on chain length.

The situation of a polymer in solution which has stiff chains is different from the case discussed above. If rupture takes place in such a chain, translational diffusion of the two chain fragments will take place. If this type of diffusion is much faster than segmental diffusion, then actual chain scission will practically be completely determined by translational diffusion out of cages. If, however, translational diffusion is much slower than segmental diffusion, chain scission will be dominated by segmental diffusion. The cage for translational diffusion has an approximate radius of a size corresponding to the radius of a chain in random coil conformation having a chain length $D P_{0}$; this radius is proportional to the square root of the chain length. The chain scission rate constant for translational diffusion, which is based on Smoluchowski's ${ }^{3}$ calculations for translational diffusion, is given by

$$
k_{\mathrm{TR} . \mathrm{D} .}=\frac{3 D_{x, y}}{R_{x, y, \mathrm{TR} . \mathrm{D}}^{2}}
$$

In this case the chain scission rate constant will be inversely proportional to the solvent viscosity and also approximately to the $3 / 2$ power of the chain length.

The kinetics of some examples of chain scission reactions is treated below.

\section{CHAIN SCISSION IN SOLUTION}

(a) Completely flexible coil; disproportionation

$$
\mathbf{P}_{x+y} \underset{k_{-1}}{\stackrel{k_{1}}{\rightleftarrows}} \text { (cage) }
$$

$$
\text { chain scission 〈cage }>\stackrel{k_{2}}{\longrightarrow} \mathbf{P}_{x}+\mathbf{P}_{y}
$$

P stands for "dead" polymer molecules. The rate of chain scission is given by

$$
-\frac{\mathrm{d}[n]_{t}}{\mathrm{~d} t}=k_{2}[\langle\text { cage }\rangle]
$$

The stationary state for cages is

$$
[\langle\text { cage }\rangle]=\frac{k_{1}}{k_{-1}+k_{2}}[n]_{0} \approx \frac{k_{1}}{k_{-1}}[n]_{0}
$$

Here $[n]_{t}$ and $[n]_{0}$ are the main chain link concentrations at time $t$ and $t=0$, respectively. Hence

$$
\begin{aligned}
-\frac{\mathrm{d}[n]_{t}}{\mathrm{~d} t} & =k_{2} \frac{k_{1}}{k_{-1}}[n]_{0}=\frac{4.14 D_{x, y} k^{\prime} \mathrm{e}^{-B}}{\bar{R}_{x, y}^{2}} \frac{k_{1}}{k_{-1}}[n]_{0} \\
& =k_{\mathrm{exp}}[n]_{0}
\end{aligned}
$$

or

$$
\alpha=\frac{1}{\overline{D P_{n, t}}}-\frac{1}{D P_{0}}=k_{\text {exp }} t
$$

$\alpha$ is the degree of degradation.

(b) Completely flexible coil; radical formation; termination by solvent, depropagation

$$
\begin{aligned}
& \mathbf{P}_{x+y} \stackrel{\stackrel{k_{1}}{\rightleftarrows} \text { cage }}{\stackrel{k_{-1}}{\longrightarrow}} \\
& \text { cage } \stackrel{k_{2}}{\longrightarrow} \mathbf{R}_{x+}+\mathbf{R}_{y} \\
& \mathbf{R}_{x}\left(\text { or } \mathbf{R}_{y}\right) \stackrel{k_{3}}{\longrightarrow} \mathbf{R}_{x-1}\left(\text { or } \mathbf{R}_{y-1}\right)+\mathrm{m}_{1} \text { etc. }
\end{aligned}
$$

fast: $\mathbf{R}+\mathbf{S} \stackrel{k_{4}}{\longrightarrow} \mathbf{P}$

$\mathrm{R}$ stands for polymer radicals and $\mathrm{m}_{1}$ and $\mathrm{S}$ for monomer and solvent, respectively. Again one has for random main chain link scission and termination by solvent, respectively

$$
\begin{aligned}
-\frac{\mathrm{d}[n]_{t}}{\mathrm{~d} t} & =k_{2}[\langle\text { cage }\rangle] \\
& =\frac{4.14 D_{x, y} k^{\prime} \mathrm{e}^{-B}}{\bar{R}_{x, y}} \frac{k_{1}}{k_{-1}}[n]_{0}=k_{\exp }[n]_{0}
\end{aligned}
$$

The rate of monomer formation from a volume 


\section{H. H. G. JELLINEK}

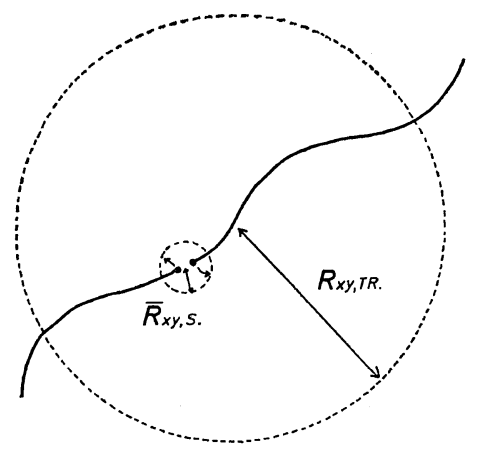

Figure 2. Chain scission of a stiff chain in solution $\left(\boldsymbol{R}_{x y, \mathrm{TR} .} \sim\right.$ cage-polymer-chain radius).

unit is given by

$$
\frac{\mathrm{d}\left[\mathrm{m}_{1}\right]}{\mathrm{d} t}=2 k_{2}[\langle\text { cage }\rangle] \bar{\varepsilon}=k_{3}[\mathrm{R}]
$$

Here $\bar{\varepsilon}$ is the number-average kinetic chain length of the depropagation reaction; $\bar{\varepsilon}$ is given by $^{4}$

$$
\bar{\varepsilon}=\frac{k_{3}}{k_{4}[\mathrm{~S}]}=\frac{k_{3}}{k_{4}{ }^{\prime}}
$$

[R] is the polymer radical concentration. The steady state concentration of the latter is

$$
[\mathrm{R}]_{\mathrm{s} . \mathrm{s} .}=\frac{2 k_{2}[\langle\text { cage }\rangle]}{k_{4}{ }^{\prime}} \frac{2 k_{2} k_{1}[n]_{0}}{k_{4}^{\prime} k_{-1}}
$$

Hence

$$
\frac{\mathrm{d}\left[\mathrm{m}_{1}\right]}{\mathrm{d} t}=\frac{2 k_{2} k_{1} k_{3}}{k_{4}{ }^{\prime} k_{-1}}[n]_{0}=k_{\exp }^{\prime}[n]_{0}
$$

The rate of monomer formation should be proportional to the reciprocal solvent viscosity but independent of chain length as long as $\bar{\varepsilon} \ll \overline{D P}_{n, t}$; however, if $\bar{\varepsilon} \gg D P$, then it is also proportional to the original chain length.

(c) Stiff polymer chains in solution

If translational diffusion is slow, then chain scission is mainly effected by segmental mobility of chain ends and the results are the same as before.

If translational diffusion is fast, one obtains for the case treated under (a)

$$
\begin{aligned}
-\frac{\mathrm{d}[n]_{t}}{\mathrm{~d} t} & =k_{2} \frac{k_{1}}{k_{-1}}[n]_{0}=\frac{3 D_{x, y}}{R_{x, y}^{2}, \text { TR.D. }} \frac{k_{1}}{k_{-1}}[n]_{0} \\
& =k_{\text {exp }}[n]_{0}
\end{aligned}
$$

$k_{\text {exp }}$ should in this case be proportional to the reciprocal solvent viscosity and to the reciprocal chain length raised to approximately the $3 / 2$ power.

For case (b), the same results as expressed in eq 15 are obtained; the rate or monomer formation is given by eq 14 with the appropriate value for $k_{2}$ (eq 5).

If translational diffusion is slow and a polymer molecule is by some means transformed from a flexible to a stiff chain, then $B$ in the exponential factor is likely to increase and the chain scission rate constant will decrease with increasing stiffness.

\section{CHAIN SCISSION IN BULK POLYMERS}

Here the medium cage will contain essentially chain end segments. It is likely that chain end segment diffusion is predominantly governed by the local medium viscosity and will not appreciably depend on polymer chain length especially if the chain length is large. If the local viscosity increases, the rate constant will decrease; the rate constant will also depend on the morphology of the polymer in addition to the effect of crystallinity on diffusion rates of reactants into the polymer. The mobility of chain and segments in crystalline polymers will be smaller than in amorphous ones. However, in certain cases crystalline fold regions will be especially susceptible to chain scission because of their increased stress lowering the energy of activation for reaction in these regions. ${ }^{5}$

\section{OVERALL ENERGY OF ACTIVATION FOR CHAIN SCISSION}

The overall energy of activation for case (a) is given by

$$
E_{\text {overall }}=E_{2}+E_{1}-E_{-1}
$$

The dominant term is $E_{1}$, representing the energy of activation for scission of main chain links; $E_{2}$, the energy of activation for diffusion out of cages (also containing the energy of activation for rotation of segments in appropriate cases), will be relatively small; also $E_{-1}$, the energy of activation for recombination of polymer fragments in cages, will be small. The 


\section{Diffusion and Random Chain Scission}

same considerations can be applied to all the and other cases.

$$
k_{1} / k_{-1}=\frac{1}{2.0 \times 10^{16}}
$$

\section{DISCUSSION}

There is very little experimental work available which has been done with the express purpose of investigating cage reactions involved in chain scission. The only work carried out from this point of view is concerned with thermal chain scission of poly $(\alpha$-methylstyrene) in solvents of various viscosities. ${ }^{1}$ However, there are also data on other polymers available which can be discussed in terms of chain scission via cages.

Thermal chain scission of poly $(\alpha$-methylstyrene $)^{1}$ was investigated in cyclohexanol, which has a relatively high viscosity, cyclohexane, and a 1:1-molar mixture of these two solvents. Random initiation, depropagation and termination take place. At appreciable conversions to monomer, the kinetic chain length becomes larger than the polymer chain length. The experimental rate constants for monomer formation are directly proportional to the reciprocal solvent viscosity. Chain scission is also found to be independent of chain length as long as $\bar{\varepsilon} \ll D P$. These results fit exactly case (b) or (c), i.e., chain scission governed by chain end segment diffusion or mobility. Actually, it was inadvertently omitted to divide the relevant rate constants in Table $\mathrm{V}$ of the paper (ref 1 ) by $\bar{R}_{x, y}^{2}$; at that time $R_{x, y}$ was taken as the cage radius for translational diffusion.

The magnitude of the rate constant

$$
k=\frac{4.14 D_{x, y}}{R_{x, y}^{2}} \sec ^{-1}
$$

for unhindered rotation can be estimated for the present case $\left(D_{x, y}=10^{-6}\right.$ to $10^{-7} \mathrm{~cm}^{2} \mathrm{sec}^{-1}$ and $\left.R_{x, y}=3 \times 10^{-8} \mathrm{~cm}\right)$. Then $k_{2}$ is about $10^{+8}$ to $10^{+9} \mathrm{sec}^{-1}$. A concentration of $0.1 \mathrm{~mol}$ of main chain links per liter and a chain length of $5 \times$ $10^{3}$ give $2 \times 10^{-5} \mathrm{~mol}$ of original chains in each liter of solution (this corresponds to the experimental conditions). If one link per chain is broken on the average in $1 \mathrm{hr}$, then

$$
\alpha=\frac{1}{5 \times 10^{3}}=10^{9} \frac{k_{1}}{k_{-1}} \times 3.6 \times 10^{3}
$$

This result indicates, as expected, that recombination of chain fragments in a cage is a very fast and frequent process, even if $k_{2}$ is multiplied by a small factor taking care of hindered segment mobility. The steady-state cage concentration can also be estimated and amounts for this case to $c a .10^{-17} \mathrm{~m} / l$ for unhindered rotation. It is reasonable that the cage concentration is so small as main chain scission is quite a rare occurrence. The total number of completed main chain scissions in one liter per hour under the above conditions is only $2 \times 10^{-5}$ mol. The effect of chain scission of this small magnitude is so noticeable because of the long chain nature of the molecules.

Some experiments concerning chain scission of atactic polystyrene by nitrogen dioxide in dioxane solution may be mentioned. Here the experimental rate constants decrease approximately inversely with the reciprocal polymer chain length. The chain lengths were two to three times smaller than those in the case of $\operatorname{poly}(\alpha$-methylstyrene). This relatively small length may be of a magnitude which affects segmental mobility.

The photolyses of atactic and isotactic poly(methacrylic acid) as a function of $\mathrm{pH}$ values are of interest. ${ }^{7}$ The photolysis rate constants for the atactic acid are independent of chain length at any one $\mathrm{pH}$ value, but are inversely proportional to the root mean square end to end distance. The latter increases linearly with $\mathrm{pH}$ values. The isotactic acid behaves similarly except that there is a slight dependence of the rate constant on molecular weight. On the whole this behavior corresponds to translational diffusion for chain scission, but the picture is obscured by effects apparently due to other causes. The chains become stiffer with increasing $\mathrm{pH}$ values or increasing ionization of the acid increasing $B$, i.e., $B=k^{\prime \prime} \mathrm{pH}$.

Photolysis of polyacrylonitrile of different chain lengths in dilute solution gives rate constants which increase with decreasing chain length. If this dependence were due to translational diffusion the rate constants should de- 
crease with increasing chain length. However, the polymer samples were prepared by several different methods.

The photolysis of poly(methyl methacrylate) in solutions of 2-chloroethanol results in almost straight line $v s$. time curves. Thus there is very little or no influence of chain length on the rate constants agreeing with segmental mobility.

During chain scission of bulk polystyrene fractions by $\mathrm{NO}_{2},{ }^{5}$ a similar dependence of rate constants on chain length as in the case of polystyrene in solution was observed. Here again the chain length may still be of magnitudes where they exert some influence on segmental mobility.

$\alpha$ vs. time plots for oxidative thermal degradation of isotactic polystyrene films give straight lines; here neither chain length nor bulk viscosity have any effect agreeing with completion of chain scission by segmental mobility. Also, photolysis of isotactic polystyrene films gives straight line $\alpha$ vs. time plots. ${ }^{8}$

In conclusion it can be stated that there is as yet very little experimental work available suitable for the evaluation of the chain scission process on the basis of diffusion of polymer fragments from cages. In the one case where such data have been obtained especially for this purpose, operation of segmental diffusion is indicated. The other cases discussed above are largely speculative. Thus, there is need for research in this area in order to understand molecular processes pertaining to chain scission.

Acknowledgments. Thanks are due to Drs. K. Horie and I. Mita for helpful discussions. The author also wishes to express his thanks to Professor H. Kambe for his hospitality and for his interest in this work.

\section{REFERENCES}

1. H. H. G. Jellinek and Ming Dean Luh, Eur. Polym. J. Suppl. 149-171 (1969).

2. S. W. Benson and A. M. North, J. Amer. Chem. Soc., 84, 935 (1962).

3. M. V. Smoluchowski, Z. Phys. Chem., 92, 129 (1918).

4. H. H. G. Jellinek, "The Encyclopedia of Science and Technology of Polymers, Vol. 4," John Wiley and Sons Inc., New York, N. Y., 1966, p 740 .

5. P. Hrdlovic, J. Pavlinec, and H. H. G. Jellinek, J. Polym. Sci. A-1, 9, 1235 (1971).

6. H. H. G. Jellinek and F. Flajsman, ibid., $A-1$, 7, 1153 (1969).

7. H. H. G. Jellinek and H. Chou, Can. J. Chem., 42, 522 (1965); H. H. G. Jellinek and S. N. Lipovac, Makromol. Chem., 1, 783 (1966); see also C. MacDonald and H. H. G. Jellinek, $J$. Colloid. Interfac. Sci., 38, 101 (1972).

8. H. H. G. Jellinek and I. J. Bastien, Can. J. Chem., 39, 2056 (1961).

9. H. H. G. Jellinek and S. N. Lipovac, Macromolecules, 3, 231, 237 (1970). 